\title{
Unconscious Activation of the Cognitive Control System in the Human Prefrontal Cortex
}

\author{
Hakwan C. Lau ${ }^{1,2}$ and Richard E. Passingham ${ }^{1,2}$ \\ ${ }^{1}$ Wellcome Department of Imaging Neuroscience, University College London, London WC1N 3BG, United Kingdom, and ${ }^{2}$ Department of Experimental \\ Psychology, University of Oxford, Oxford OX1 3UD, United Kingdom
}

\begin{abstract}
Using functional magnetic resonance imaging, we tested whether unconscious information can influence the cognitive control system in the human prefrontal cortex. Volunteers had to prepare to perform either a phonological judgment or a semantic judgment on an upcoming word, based on the instruction given at the beginning of each trial. However, in some trials they were visually primed to prepare for the alternative (i.e., "wrong") task, and this impaired their performance. This priming effect is taken to depend on unconscious processes because the effect was present even when the volunteers could only discriminate the identity of the primes at chance level. Furthermore, the effect was stronger when the visibility of the prime was near zero than when the visibility of the prime was significantly higher. When volunteers were unconsciously primed to perform the alternative task, there was also decreased neural activity in the brain areas relevant to the instructed task and increased neural activity in the brain areas relevant to the alternative task, which shows that the volunteers were actually engaged in the wrong task, instead of simply being distracted. Activity in the mid-dorsolateral prefrontal cortex was also found to be associated with this unconscious priming effect. These results suggest that the cognitive control system in the prefrontal cortex is not exclusively driven by conscious information, as has been believed previously.
\end{abstract}

Key words: consciousness; fMRI; cognitive control; priming; metacontrast masking; taskset

\section{Introduction}

Traditionally, higher cognitive control functions are thought to require consciousness and attention (Norman and Shallice, 1986; Jack and Shallice, 2001). Although stimuli presented subliminally can prime specific motor responses (Dehaene and Naccache, 2001), it is assumed that such stimuli cannot influence which task the brain should prepare to do. This operation of establishing a particular taskset is an example of the highest form of cognitive control (Miller and Cohen, 2001; Koechlin et al., 2003) and is associated with activations in the prefrontal cortex (Sakai and Passingham, 2003, 2006). The prefrontal cortex is believed to form part of a network for conscious processes (Jack and Shallice, 2001; Rees et al., 2002; Dehaene et al., 2003a). Typically, subliminally presented stimuli do not activate this system (Badgaiyan, 2000; Rees et al., 2002).

However, recent empirical findings in psychology (Bargh and Ferguson, 2000; Ferguson and Bargh, 2004; Dijksterhuis et al., 2006) suggest that a great deal of complex cognitive behavior could take place without conscious attention. Inspired by recent advances in neuroimaging research that also draw attention to the strength and depth of subliminal priming (Dehaene et al., 1998; Hannula et al., 2005; Naccache et al., 2005; Gaillard et al., 2006), we attempted to create a situation in which subliminally

\footnotetext{
Received 0ct. 4, 2006; revised April 12, 2007; accepted April 13, 2007.

This work was supported by the Wellcome Trust.

Correspondence should be addressed to Hakwan Lau, Functional Imaging Laboratory, 12 Queen Square, London WC1N 3BG, UK. E-mail: hakwan@gmail.com.

DOI:10.1523/JNEUROSCI.4335-06.2007

Copyright $\odot 2007$ Society for Neuroscience $\quad$ 0270-6474/07/275805-07\$15.00/0
}

presented stimuli influence the establishment of tasksets. This constitutes a strong test for the traditional assumption that higher cognitive functions require consciousness (Jack and Shallice, 2001). We performed a behavioral test to see whether such "unconscious" priming was possible and went on to investigate whether it involved the activation of the cognitive control system in the prefrontal cortex.

\section{Materials and Methods}

Psychological task. Volunteers were presented with words (maximum height, $1^{\circ}$ of visual angle) and were required to perform either a semantic judgment (whether the word refers to concrete objects) or a phonological judgment (whether the word is bisyllabic) (Fig. 1). The volunteers gave "yes" responses by pressing a key with their right index fingers and "no" by their right middle fingers, and they had to press the keys within $2 \mathrm{~s}$ after the onset of the word. Importantly, the volunteers were only instructed which task they were to perform at the beginning of each trial, shortly $(150 \mathrm{~ms})$ before they saw the target word. Therefore, they had to prepare the taskset quickly once they saw the instructions.

These instructions were coded by two arbitrary shapes (visual angle, $1.5 \times 1.5^{\circ}$ ): a square meant the phonological task, and a diamond (i.e., a square tilted by $45^{\circ}$ ) meant the semantic task. Shortly before the presentation of the instruction figure, however, a smaller figure (the prime; visual angle, $1 \times 1^{\circ}$ ) was presented, to which volunteers were not asked to pay any attention. Like the instruction figure, the prime could either be a square or a diamond. On half of the trials, the prime was congruent with the instruction stimulus, and on half it was incongruent. It has been shown that the presentation of these prime figures can effectively influence the behavior of the volunteers (Mattler, 2003; Vorberg et al., 2003), presumably because they are of shapes similar to those in the instructions.

The advantage of using the semantic and phonological tasks is that we 
know from previous studies (Devlin et al., 2003; Sakai and Passingham, 2006) that they are associated with activity in distinctly different brain areas. Therefore, using functional magnetic resonance imaging (fMRI), we could directly test whether the volunteers were actually engaged in the irrelevant (i.e., uninstructed) task when the prime was incongruent to the instruction. If this was so, we should find activation in the irrelevant brain areas when the volunteers were primed to do the irrelevant task.

The advantage of the type of figures we used for the instructions and primes (Mattler, 2003; Vorberg et al., 2003) is that the instruction figures act as a metacontrast mask to the prime figures and thereby reduce the visibility of the prime (Breitmeyer, 1984). When the temporal distance between the prime and the instruction [the stimulus onset asynchrony (SOA)] was at $83 \mathrm{~ms}$, the visibility of the prime was nonsignificantly different from zero, as revealed by a forced-choice discrimination task performed after the experiment. One could therefore argue that if there was interference on incongruent trials, the priming effect was unconscious.

However, this interpretation suffers from a problem that affects almost all previous research on subliminal priming. This is the fact that it is difficult to demonstrate statistically that visibility is exactly zero (Hannula et al., 2005). The problem is that the failure to find a significant difference from zero may simply reflect the lack of statistical power because of insufficient observations or an insufficient number of subjects. Therefore, in this experiment, we adopted an additional measure to establish that the priming effect does not depend on a single conscious system. This depends on a recently developed approach (Vorberg et al., 2003; Schmidt and Vorberg, 2006), which is to try to demonstrate a double dissociation between visibility and the strength of the priming effect. If we could show that the priming effect decreased even when the visibility of the prime increased, we can confidently conclude that this priming effect cannot be driven by a single conscious process (Schmidt and Vorberg, 2006). We therefore included conditions in which the SOA was at 16 $\mathrm{ms}$. This condition yields a higher visibility when compared with the condition in which the SOA is at $\sim 83 \mathrm{~ms}$. As revealed in previous experiments on motor (Vorberg et al., 2003) and nonmotor (Mattler, 2003) priming, at short SOAs, the priming effects were generally weaker, presumably because there was less time for the prime to exert its influence. However, the visual energy of the prime is not lower at short SOAs, because of the special property of nonmonotonicity in metacontrast masking functions (Breitmeyer, 1984): when visibility is plotted against SOAs, it is usually $\mathrm{U}$-shaped in metacontrast masking.

For simplicity, we call the conditions associated with the two SOAs the High-Visibility (short SOA) and Low-Visibility conditions (long SOA). This is also justified empirically by the psychophysical results. Together with the factors of Task (Phonological vs Semantic) and primeinstruction Congruency (Congruent vs Incongruent), this constitutes a $2 \times 2 \times 2$ factorial design, which is summarized in Table 1 . The trials were paced such that there was one trial every $4 \mathrm{~s}$. Between trials, a central fixation cross was presented. There were 40 trials for each condition, and they were intermixed in a random manner.

The words used in the experiment were taken from the online version of the Medical Research Council Psycholinguistic Database (http://www.psy.uwa.edu.au/mrcdatabase/uwa_mrchtm), using $>550$ (concrete) and $<350$ (abstract) on the concreteness scale as criteria for word selection into the two semantic groups. For the phonological task, words were considered bisyllabic if they contain two syllables according to the data-
Table 1. Task design

\begin{tabular}{lll}
\hline & Task & \\
\hline Congruency & Phonological & Semantic \\
& Congruent & Congruent \\
& Phonological & Semantic \\
& Incongruent & Incongruent \\
\hline
\end{tabular}

Volunteers could be instructed to perform either the Phonological task or the Semantic task, and the prime in each trial could either be Congruent or Incongruent with the instruction. These were repeated for the conditions in which the primes were of either High Visibility or Low Visibility. Together, these constitute a $2 \times 2 \times 2$ factorial design, with Task, Congruency, and Prime Visibility as experimental factors.

base, and the non-bisyllabic group consisted of words that are either monosyllabic or trisyllabic. For every one of the eight conditions, half of the trials required the same motor response in both the phonological and the semantic tasks, and half of the trials required incompatible motor responses. These words were allocated to volunteers in a systematically counterbalanced manner, in that words that were used in the Congruent conditions in half of the volunteers were used in the Incongruent conditions in the other half. And words that were used in the High-Visibility conditions in half of the volunteers were used in the Low-Visibility conditions in the remaining half.

Data acquisition. Twelve volunteers participated in this study, and they all gave informed written consent. Data from two volunteers were, however, discarded because the manipulation of prime visibility failed for them (see below), and therefore the main analyses involved 10 volunteers. After they were told the instructions of the tasks, the volunteers practiced the tasks for $\sim 5 \mathrm{~min}$ on a computer. They then performed the tasks in the MRI scanner, where they viewed the screen through inverting lens goggles.

The functional imaging was conducted with a Siemens (Erlangen, Germany) Allegra 3.0T scanner to acquire gradient echo T2*-weighted echo- 
planar images (EPIs) with blood oxygenation level-dependent (BOLD) contrast as an index of local increases in synaptic activity. The image parameters used were as follows: matrix size, $64 \times 64$; voxel size, $3 \times 3$ $\mathrm{mm}$; echo time, $40 \mathrm{~ms}$; repetition time, $2600 \mathrm{~ms}$. A functional image volume comprised 40 contiguous slices of $2 \mathrm{~mm}$ thickness (with a $1 \mathrm{~mm}$ interslice gap), which ensured that the whole brain was within the field of view. For each participant, the experiment was divided into four runs, between which the participants were allowed a short rest and minor adjustments of their head positions to ensure comfort. In the middle of the run, i.e., after 40 trials, there was also a $30 \mathrm{~s}$ rest period. For each of these runs, we collected 153 EPI volumes.

Data analyses. The fMRI data were analyzed using the SPM2 software [Wellcome Department of Cognitive Neurology, London, UK; detailed description of the theoretical aspects can be found at http://www.fil. ion.ucl.ac.uk/spm/doc/books/hbf2/ or Frackowiak et al. (2005)]. The first six scans of the EPI series for each run were excluded from the analysis to minimize T1 relaxation artifacts. A mean image for all scan volumes was created, to which individual volumes were spatially realigned by using rigid body transformation. Unwarping was performed during realignment to correct for dynamic motion-distortion interaction artifacts. Then, the EPIs were normalized to the standard EPI template produced by the Montreal Neurological Institute, which is included in the SPM2 package. The resulting images were subsampled into a resolution of $2 \times 2 \times 2 \mathrm{~mm}$. A Gaussian filter of $10 \mathrm{~mm}$ full-width at half-maximum was then applied to smooth the data spatially, to take into account the anatomical variability between participants. The time series data at each voxel were processed using a high-pass filter with a cutoff of $128 \mathrm{~s}$ to remove low-frequency drifts. Short-term serial correlations in the data were modeled by an auto-regressive process with white noise. To reduce the impact of potential artifacts such as spikes or slice drop-out, for each volume, the signal intensity in each voxel was also normalized to the global mean of the scan.

The subject-level statistical analyses were performed using the general linear model for regression. The trials were classified into eight event types with respect to the task conditions, and the onsets of the trials were modeled with stick functions. These are convolved with the canonical hemodynamic response function specified in SPM2 to form the regressors. The parameter estimates for each regressor represent the size of the effect for each condition, and these were estimated for each voxel across the whole brain. The magnitude of these estimates reflect the maximum height of the fitted hemodynamic response function, which is in the scale of percentage signal difference at that particular voxel, relative to the global signal intensity for the whole volume at the time of the peak. We adopted standard fMRI convention and labeled these "\% BOLD signal change" in Figures 3 and 4. When the effects of the conditions were compared, the contrasts of the relevant parameter estimates were computed for each voxel to form contrast images.

To identify the peak voxels related to the Phonological and Semantic tasks, respectively, contrasts were created to compare (1) the Phonological Congruent conditions against baseline and (2) the Semantic Congruent conditions against baseline. Only the Congruent conditions were considered because in the Incongruent trials, the volunteers might be performing the irrelevant task because of the priming effect. The areas were located by taking the coordinates from a previously published study that used similar tasks (Sakai and Passingham, 2006) and navigating to the nearest peak under the relevant contrast for each subject. The parameter estimates were extracted at these coordinates to form Figure 3. This method of extracting data from each individual's peak voxel was intended to control for minor variation in individual anatomy. For the three areas in which data were extracted, i.e., the SEs across subjects for the peak coordinates $(x, y, z$; in millimeters) were 2.31, 2.04, 3.05 (left ventral premotor cortex); 1.25, 8.66, 2.43 (middle temporal gyrus); and $1.90,1.81,4.29$ (left inferior prefrontal). They suggest that the individual variability of the peak coordinates was not large, yet not negligible.

To test the effect of Congruency and the interaction between Congruency and Visibility across the whole brain, group-level $t$ tests were performed on contrast images. The statistical results were thresholded at $p<$ 0.001 uncorrected and overlayed on the standard "Colin27" brain (Montreal Neurological Institute) to produce Figure 4. However, results were

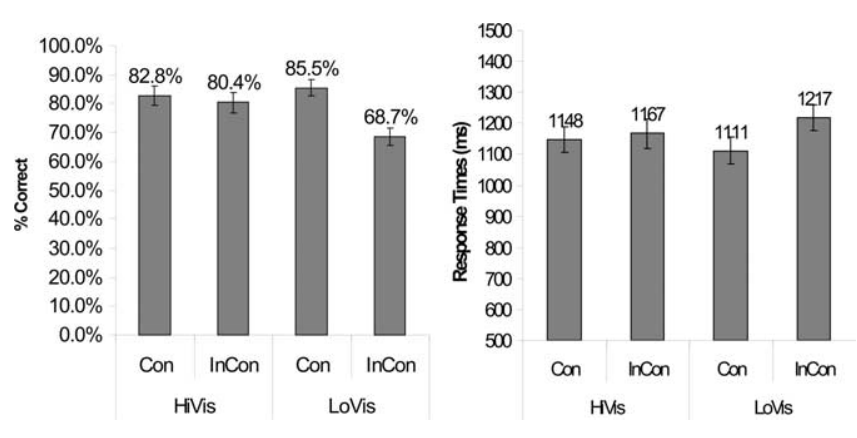

Figure 2. Behavioral demonstration of the priming effect. HiVis and LoVis, respectively, refer to the high and low visibility of the primes. Con and InCon refer to the congruency and incongruency between the primes and the instructions. When the primes were incongruent with the instructions, volunteers were primed to perform the alternative (i.e., wrong) task, and their accuracies were lower (left) and response times bigger (right). Interestingly, this was particularly true when the visibility of the primes was low, as reflected by interactions between the factors of Congruency and Visibility in both the accuracies $(p=0.001)$ and the response times $(p<0.0005)$. This justifies the claim that the priming effect operates fundamentally at an unconscious level.

only reported if either they survived a threshold of cluster-level wholebrain correction or small-volume correction when an a priori hypothesis was available [as in the case of the mid-dorsolateral prefrontal cortex (DLPFC), where the coordinates were taken from a recent study conducted by the same authors (Lau and Passingham, 2006)].

\section{Results}

\section{Psychophysics}

The data for the Phonological and Semantic conditions combined are presented in Figure 2. There was a clear interaction between the factors of Visibility and Congruency (ANOVA, $p=$ 0.001 ), in that the difference between incongruent trials and congruent trials was bigger when the prime was less visible $(p<$ $0.0005, t$ test) and was smaller and nonsignificant when the prime was more visible ( $p<0.025, t$ test). This pattern was also true when either the Phonological or Semantic conditions was considered alone (ANOVA, $p=0.009$ and 0.008 , respectively). Overall, the accuracies were not as high as expected, but this may not mean that the volunteers were performing poorly. This is because some of the words used afford equivocal answers. For instance, the word "library" is considered bisyllabic by some and trisyllabic by others, and the word "country" is considered concrete by some and abstract by others. This somewhat decreases the overall accuracies, because the answers given by the volunteers may not be considered correct by the computer program even when the volunteers were doing the tasks properly. However, because the words were randomly assigned to different conditions, with the assignment counterbalanced across subjects, the relative difference in the accuracies should still reflect different difficulties.

The response times were calculated only based on the correct trials. The data for the Phonological and Semantic conditions combined are presented in Figure 2. As with the accuracies, there was a clear interaction between the factors of Visibility and Congruency (ANOVA, $p=0.01$ ), in that the difference between incongruent trials and congruent trials was bigger and significant when the prime was less visible $(p<0.0005, t$ test $)$ and was smaller and nonsignificant when the prime was more visible ( $p=$ $0.106, t$ test). This effect was also significant for the Phonological conditions (ANOVA, $p<0.0005$ ) but not for the Semantic conditions because the variances were larger.

After the main experiment, volunteers were further tested in the scanner to see whether the visibility of the primes at SOAs 
chosen for the High-Visibility and LowVisibility conditions really differed. It was explained at this point that before the presentation of the instruction, a smaller figure (i.e., the prime) was presented; this had not been mentioned before scanning. The volunteers were now presented again with the stimuli and asked to pay full attention to this smaller figure and press keys on every trial to say whether it was a square or a diamond. The SOAs were either 16 or $83 \mathrm{~ms}$, as in the High- and LowVisibility conditions, respectively, as in the main experiment.

Overall, the volunteers performed better at an SOA of 16 ms. However, two volunteers performed better at an SOA of 83 ms over an SOA of $16 \mathrm{~ms}$ by a margin of $>5 \%$ in their accuracies. The data of these two volunteers were therefore discarded from all other analyses because this means that they were likely to have perceived primes better in the Low-Visibility condition than in the High-Visibility condition, thus violating the logic of the study.

For the remaining 10 volunteers, at an SOA of $83 \mathrm{~ms}$, which was the same for the Low-Visibility conditions, the averaged performance (and the SD across subjects), as measured by the bias-free measure $d^{\prime}$ (Macmillan and Creelman, 1991), was 0.05 ( $\mathrm{SD}=0.33)$. This is not significantly larger than zero $(p=0.32)$. At an SOA of $16 \mathrm{~ms}$, which was the same for the HighVisibility conditions, the averaged performance (and the SD across subjects), as measured by the bias-free measure $d^{\prime}$, was 0.26 ( $\mathrm{SD}=0.33)$. As predicted, this was significantly higher than the performance at an SOA of $83 \mathrm{~ms}$ ( $p=0.04$, paired $t$ test). Overall, these data suggest that the primes in the LowVisibility conditions were not significantly visible and more importantly were significantly less visible than the primes in the High-Visibility condition.

To further ensure that the priming effect in the Low-Visibility condition was not caused by the conscious perception of the primes, we also assessed the relationship between the visibility of the primes and the size of the priming effect in this condition. We adopted the procedure advocated by Greenwald et al. (1996), which involves performing linear regression to try to predict the size of the priming effects from the visibility of the primes. We performed this on both the reaction time effects and the accuracies and found that in both cases, the vertical intercepts for the regression models were significantly positive. For the reaction time effect (the size of increase from Congruent to Incongruent), this was $107.35 \mathrm{~ms}(p<0.0005)$. For the accuracy effect (the size of decrease from Congruent to Incongruent), this was $17.8 \%$ $(p<0.0005)$. These results imply that if we were to interpolate from the data, when visibility is exactly at zero (the vertical intercepts), the predicted priming effects would still be significantly positive. This supports the claim that in the Low-Visibility condition, the priming effect did not depend on the conscious perception of the primes.
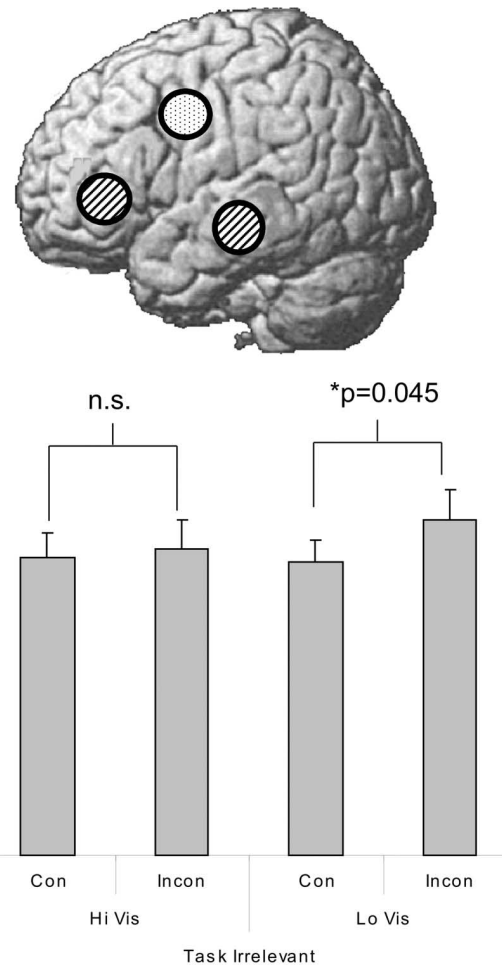

Task Relevant
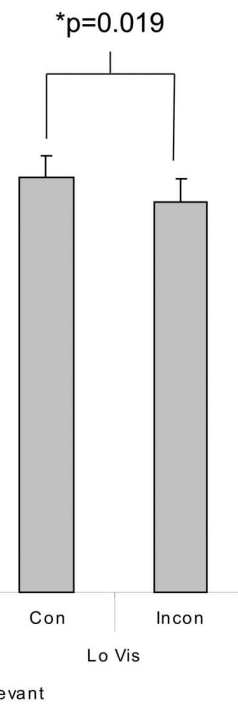

Figure 3. Neural activity associated with priming. Data were extracted from the brain areas that were previously found to be associated with the Phonological task (left ventral premotor area) and the Semantic task (left inferior frontal cortex and middle temporal gyrus). The location of these is schematically illustrated on the brain above. Here, task relevant means activity from the whe Phonological task. Task irrelevant means the activity was extracted from the alternative areas, 列 (see Fig. 2), activity in task-relevant areas was significantly reduced ( $p=0.019)$, and f the prime. This is reflected by a three-way interaction between Task Relevance (i.e., activity in task relevant areas vs activity in task irrelevant areas), Congruency (Con; between prime and instruction), and Visibility (of the prime) $(p=0.040)$. InCon, Incongruency; n.s., not significant.

\section{Brain imaging}

The behavioral data show that when the visibility of the prime was low, there was a stronger priming effect. We therefore analyzed the fMRI data to test whether this effect was associated with changes in the level of activity in the task-specific brain areas. From previous studies (Devlin et al., 2003; Sakai and Passingham, 2006), we know that the Phonological task should be associated with activity in the left ventral premotor cortex, and the Semantic task should be associated with activity in the left middle temporal gyrus and the left inferior prefrontal. Therefore, we extracted the data from these areas for each volunteers and tested whether the priming effect was associated with activity in the task-relevant areas on congruent trials and task-irrelevant areas on incongruent trials.

The data for the "task relevant" activity are shown in Figure 3 (left). Here, task relevant means activity in the left middle temporal gyrus and the left inferior prefrontal cortex when the Semantic task is considered and activity in the left ventral premotor area when the Phonological task is considered. As shown in the figure, in the Low-Visibility condition, the neural activity was lower when the primes were incongruent than when they were congruent to the instruction. This suggests that when volunteers were primed to do a different task, there was less involvement of 

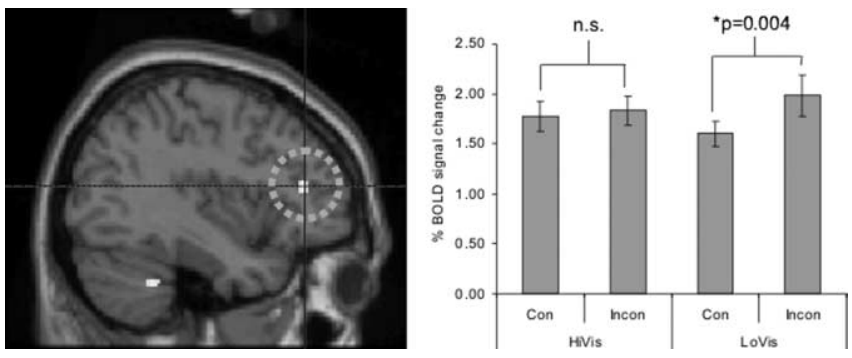

Figure 4. Mid-DLPFC and unconscious priming. We looked for activity in the brain that was associated with the unconscious priming effect in general, regardless of which task was explicitly cued, by testing for the interaction between Congruency (Con; between prime and instruction) and Visibility (of the prime). This test revealed activity in the mid-DLPFC (right; $x=-38$ ). This effect was specific to the Low-Visibility condition, in which volunteers did not consciously perceive the primes. InCon, Incongruency; n.S., not significant; HiVis, high visibility; LoVis, low visibility.

the task-relevant areas ( $p=0.019, t$ test). It is important to note, however, that this effect was not significant when the visibility of the prime was high $(p=0.146, t$ test $)$.

The data for the task-irrelevant activities are also shown in Figure 3 (right). Here, "task irrelevant" means activity in the left middle temporal gyrus and the left inferior prefrontal cortex when the volunteers were instructed to perform the Phonological task and activity in the left ventral premotor area when the volunteers were instructed to perform the Semantic task. As shown in the figure, in the Low-Visibility condition, the neural activity was higher in task-irrelevant areas when the primes were incongruent than when they were congruent with the instruction. This suggests that when volunteers were primed to perform a different task, there was more involvement of the areas relevant for the primed task instead of the explicitly instructed task ( $p=0.045, t$ test). Again, it is important to note that this effect was not significant when the visibility of the prime was low $(p=0.268, t$ test $)$.

Overall, the opposite effects observed for the task-relevant and task-irrelevant activity was reflected in a significant three-way interaction between the factors of Visibility, Congruency, and the Relevance of the activity to the task (Relevant vs Irrelevant) (ANOVA, $p=0.040$ ).

We finally tested whether activity in the prefrontal cortex was associated with the priming effect. We did this by considering the Phonological and Semantic conditions together. The main effect of Congruency revealed activity in the medial frontal cortex (coordinates: $-10,46,16 ; p<0.0005$, cluster-level whole-brain corrected): the peak lay on the borders between the paracingulate sulcus and the pre-supplementary motor area. However, activity in this region showed no interaction between Congruency and Visibility, which means the priming effect observed here was not necessarily specific to unconscious processes.

The test of interaction between Congruency and Visibility revealed activity in the mid-dorsolateral prefrontal cortex (Fig. 4, right) (coordinates: $-38,36,14 ; p<0.001$, uncorrected; $p<0.05$ under small volume correction). The interaction was in the direction that the priming effect increased as visibility decreased, as with the results of the psychophysics. In other words, when the visibility of the primes was low, the difference in activity between the incongruent condition and the congruent condition was significant ( $p=0.004, t$ test). This effect was, however, nonsignificant $(p=0.206, t$ test $)$ when the visibility of the primes was high.

\section{Discussion}

The effects of subliminal priming have traditionally been thought to be limited in scope (Hannula et al., 2005). In fact, some psy- chophysicists still believe that many reported subliminal priming effects can be explained away as artifacts (Holender and Duscherer, 2004). The major problem is in establishing that the primes are in fact invisible rather than faintly visible. In this experiment, we demonstrated in the standard way that the priming effect was present even when the visibility of the primes was low and nonsignificantly different from zero. We further showed that in the regression models in which the visibility of the primes was used to predict the priming effect, the vertical intercepts were significantly positive. This means that if we theoretically interpolate based on the data, when the visibility of the primes is at exactly zero, we should still predict significantly positive priming effects. On top of these standard criteria for demonstrating unconscious priming, we have additionally used a recently developed approach to ensure that the priming effect is unconscious. We managed to show that the priming effect and visibility could be altered in different directions; that is, when the visibility of the prime was low and close to zero, the priming effect was stronger than when there was significant visibility. Whereas this does not imply that the decrease in prime visibility is the cause of the increase in priming effect, the fact that the two can change in different directions constitutes a double dissociation, which formally rejects the hypothesis that the priming effect is the result of a single conscious process (Schmidt and Vorberg, 2006). In other words, one can infer from the data that the priming effect depends positively on a process that neither contributes to nor correlates with the visibility of the primes. Within the current context, one could consider this process unconscious (in regard to the perception of the primes).

This approach of double dissociation has previously been used to demonstrate unconscious motor priming using psychophysics (Vorberg et al., 2003). We have extended the finding in three ways. First, we have shown that subliminal primes can influence the establishment of an arbitrary and large taskset, thus influencing cognitive control. Second, we have used imaging to demonstrate that subliminal priming can give rise to task-irrelevant neural activity when the prime and the instruction are incongruent. Finally, and most importantly, we have used imaging to show that activity in the mid-dorsolateral prefrontal cortex is associated with the priming effect. These findings, together with previous research on the depth of subliminal priming (Dehaene et al., 1998; Hannula et al., 2005; Naccache et al., 2005; Gaillard et al., 2006), challenge critics to reconsider the limits of unconscious processing.

Using a similar task paradigm, Mattler (2003) has also reported the subliminal priming of different "mental operations." In one experiment, the volunteers were primed to perform a task that was incompatible with the task that was explicitly instructed, as in the present experiment. The finding was that in this condition, the volunteers were slower to respond. However, because no neural activity was recorded, it could be argued that the volunteers were slower simply because they were distracted by the lack of congruency of the visual stimuli. Alternatively, the distraction could operate at a higher cognitive level, yet the volunteers were only distracted in general but not engaged actively in the wrong task.

In this experiment, we resolved this issue by using fMRI. We demonstrated that the priming effect was present because volunteers were actually engaged in the irrelevant task operations. We could do this because we deliberately used tasks that are known to involve spatially different brain areas. We found that when volunteers were primed to perform an incongruent task, activity in the brain areas responsible for the instructed task decreased, and 
activity in the brain areas responsible for the primed task increased. This effect was significant only when the primes were less visible. This constitutes a strong demonstration that the priming effect operates at a task-specific level, that is that the volunteers were actually engaged in the irrelevant task operations when primed.

Another important feature of our experiment is that, unlike the simple tasks used by Mattler (2003) that involved a stimulus set of 4 , our tasks afforded a virtually infinite set of stimuli, and that a new unpredictable word was be presented in every trial. After seeing the task instruction, the volunteers had to react to the challenge of preparing an abstract task set that was not restricted to a simple limited set of stimulus-response mappings. It is interesting that even at this level of task complexity, the cognitive control system could be influenced unconsciously.

The subliminal priming effect was associated with activity in the mid-DLPFC (Brodmann's area 46) (Petrides and Pandya, 1999). Again, we interpret this effect as subliminal because it was found by testing the interaction of the factors of Congruency and Visibility. The difference in the level of activity between incongruent primes and congruent primes was low and nonsignificant when the primes were visible, but it was significant when the visibility of the primes was not significantly different from zero. This is compatible with the observation that the establishment of tasksets or goals depends on activity in the prefrontal cortex (Sakai and Passingham, 2003, 2006).

The mid-DLPFC may have been activated because, when the prime was incongruent with the instruction, the volunteers were led to prepare for two task sets instead of one, and this increased the cognitive demands. Alternatively, it could be that incongruency induced a high level of cognitive conflict, and activity in the mid-DLPFC was driven by a conflict signal project from another area (Botvinick et al., 2001, 2004). It has been reported that cognitive conflict induces activity in both the mid-DLPFC and the medial frontal cortex (MacDonald et al., 2000; Botvinick et al., 2001, 2004). The current data do not distinguish these two hypotheses, partly because activation in an area does not necessarily reflect increase in neuronal firing, or the output signal to other areas; an activation could be driven by an increase in the synaptic input (Norris, 2006).

Dehaene et al. (2003b) have reported that when stimuli causing the conflict were masked and thus rendered unconscious, activity in the medial frontal cortex no longer differentiated the levels of conflict. Compatible with this observation, we did not observe the subliminal priming effect in the medial frontal cortex. This was true, although we did find activity in the medial frontal cortex when we compared trials with incongruent primes with trials with congruent trials, regardless of the visibility of the primes.

Whatever the explanation of the activity in the mid-DLPFC, we can conclude that this higher cognitive area can be influenced by unconscious information. This is an interesting result because traditionally higher cognitive control functions are considered to require consciousness and attention (Norman and Shallice, 1986; Jack and Shallice, 2001). Activations in the mid-DLPFC have been associated with attentionally demanding tasks (Duncan and Owen, 2000; Lau et al., 2004) and conscious visual perception (Lau and Passingham, 2006). It has also been proposed that this area forms part of a network for conscious processing (Jack and Shallice, 2001; Rees et al., 2002; Dehaene et al., 2003a).

Our results refute the claim that the mid-DLPFC is exclusive to conscious processing (Badgaiyan, 2000). Some previous reports are in line with our results. Prefrontal activity has been reported in an implicit motor learning study, although the activity was only significant when a liberal threshold was used, and at that threshold, there was also activation in the visual cortex (Rauch et al., 1997). Moutoussis and Zeki (2002) have described a binocular masking paradigm (called binocular fusion) in which prefrontal activity was found to be higher when the volunteers did not see the presented figures. It could be argued that the volunteers were trying harder to see the stimuli when they were masked, and it is this increased effort that was reflected by the prefrontal activity. However, despite these possible criticisms of the studies, when taken together with the results reported here, the converging evidence supports that activity in the mid-DLPFC is not exclusive to conscious processes.

One of the limitations of the present study is that the volunteers were actively engaged in identifying the instruction. It remains to be seen whether the prefrontal cortex and the cognitive control system can be activated entirely unconsciously, without active attention. Also, the priming effect described here has only been shown to last for a short period of time (i.e., the time between the priming and the target word, which was either 166 or $233 \mathrm{~ms}$ ). It has been reported that a similar priming effect could not last for $>300 \mathrm{~ms}$ (Mattler, 2005). For this reason, at the temporal resolution of fMRI, we cannot currently distinguish whether the unconscious information induced sustained activity that reflects task preparation or transient activity that reflects task execution. In the past, we have made such distinction using fMRI (Sakai and Passingham, 2003, 2006), but the technique requires a variable delay between the cue and the target words in the order of $\sim 10 \mathrm{~s}$. It remains an open question whether there would be a future experiment paradigm in which an unconscious effect could be shown to last for that amount of time. However, although these possibilities may seem unlikely, it may only take a single future study to reject the null hypothesis that they cannot occur.

\section{References}

Badgaiyan RD (2000) Executive control, willed actions, and nonconscious processing. Hum Brain Mapp 9:38-41.

Bargh JA, Ferguson MJ (2000) Beyond behaviorism: on the automaticity of higher mental processes. Psychol Bull 126:925-945.

Botvinick MM, Braver TS, Barch DM, Carter CS, Cohen JD (2001) Conflict monitoring and cognitive control. Psychol Rev 108:624-652.

Botvinick MM, Cohen JD, Carter CS (2004) Conflict monitoring and anterior cingulate cortex: an update. Trends Cogn Sci 8:539-546.

Breitmeyer BG (1984) Visual masking: an integrative approach. Oxford: Oxford UP.

Dehaene S, Naccache L (2001) Towards a cognitive neuroscience of consciousness: basic evidence and a workspace framework. Cognition 79:1-37.

Dehaene S, Naccache L, Le Clec'H G, Koechlin E, Mueller M, DehaeneLambertz G, Van de Moortele PF, Le Bihan D (1998) Imaging unconscious semantic priming. Nature 395:597-600.

Dehaene S, Sergent C, Changeux JP (2003a) A neuronal network model linking subjective reports and objective physiological data during conscious perception. Proc Natl Acad Sci USA 100:8520-8525.

Dehaene S, Artiges E, Naccache L, Martelli C, Viard A, Schurhoff F, Recasens C, Martinot ML, Leboyer M, Martinot JL (2003b) Conscious and subliminal conflicts in normal subjects and patients with schizophrenia: the role of the anterior cingulate. Proc Natl Acad Sci USA 100:13722-13727.

Devlin JT, Matthews PM, Rushworth MF (2003) Semantic processing in the left inferior prefrontal cortex: a combined functional magnetic resonance imaging and transcranial magnetic stimulation study. J Cogn Neurosci 15:71-84.

Dijksterhuis A, Bos MW, Nordgren LF, van Baaren RB (2006) On making the right choice: the deliberation-without-attention effect. Science 311:1005-1007. 
Duncan J, Owen AM (2000) Common regions of the human frontal lobe recruited by diverse cognitive demands. Trends Neurosci 23:475-483.

Ferguson MJ, Bargh JA (2004) How social perception can automatically influence behavior. Trends Cogn Sci 8:33-39.

Frackowiak RSJ, Friston K, Frith C, Dolan R, Price CJ, Zeki S, Ashburner J, Penny W (2005) Human brain function. London: Academic.

Gaillard R, Del Cul A, Naccache L, Vinckier F, Cohen L, Dehaene S (2006) Nonconscious semantic processing of emotional words modulates conscious access. Proc Natl Acad Sci USA 103:7524-7529.

Greenwald AG, Draine SC, Abrams RL (1996) Three cognitive markers of unconscious semantic activation. Science 273:1699-1702.

Hannula DE, Simons DJ, Cohen NJ (2005) Imaging implicit perception: promise and pitfalls. Nat Rev Neurosci 6:247-255.

Holender D, Duscherer K (2004) Unconscious perception: the need for a paradigm shift. Percept Psychophys 66:872-881.

Jack AI, Shallice T (2001) Introspective physicalism as an approach to the science of consciousness. Cognition 79:161-196.

Koechlin E, Ody C, Kouneiher F (2003) The architecture of cognitive control in the human prefrontal cortex. Science 302:1181-1185.

Lau HC, Passingham RE (2006) Relative blindsight in normal observers and the neural correlate of visual consciousness. Proc Natl Acad Sci USA 103:18763-18768.

Lau HC, Rogers RD, Ramnani N, Passingham RE (2004) Willed action and attention to the selection of action. NeuroImage 21:1407-1415.

MacDonald AW3, Cohen JD, Stenger VA, Carter CS (2000) Dissociating the role of the dorsolateral prefrontal and anterior cingulate cortex in cognitive control. Science 288:1835-1838.

Macmillan N, Creelman C (1991) Detection theory: a user's guide. Cambridge, UK, New York: Cambridge UP.

Mattler U (2003) Priming of mental operations by masked stimuli. Percept Psychophys 65:167-187.

Mattler U (2005) Inhibition and decay of motor and nonmotor priming. Percept Psychophys 67:285-300.
Miller EK, Cohen JD (2001) An integrative theory of prefrontal cortex function. Annu Rev Neurosci 24:167-202.

Moutoussis K, Zeki S (2002) The relationship between cortical activation and perception investigated with invisible stimuli. Proc Natl Acad Sci USA 99:9527-9532.

Naccache L, Gaillard R, Adam C, Hasboun D, Clemenceau S, Baulac M, Dehaene S, Cohen L (2005) A direct intracranial record of emotions evoked by subliminal words. Proc Natl Acad Sci USA 102:7713-7717.

Norman DA, Shallice T (1986) Attention to action: willed and automatic control of behavior. In: Consciousness and self-regulation (Davidson RJ, Schwartz GE, Shapiro D, eds), New York: Plenum.

Norris DG (2006) Principles of magnetic resonance assessment of brain function. J Magn Reson Imaging 23:794-807.

Petrides M, Pandya DN (1999) Dorsolateral prefrontal cortex: comparative cytoarchitectonic analysis in the human and the macaque brain and corticocortical connection patterns. Eur J Neurosci 11:1011-1036.

Rauch SL, Whalen PJ, Savage CR, Curran T, Kendrick A, Brown HD, Bush G, Breiter HC, Rosen BR (1997) Striatal recruitment during an implicit sequence learning task as measured by functional magnetic resonance imaging. Hum Brain Mapp 5:124-132.

Rees G, Kreiman G, Koch C (2002) Neural correlates of consciousness in humans. Nat Rev Neurosci 3:261-270.

Sakai K, Passingham RE (2003) Prefrontal interactions reflect future task operations. Nat Neurosci 6:75-81.

Sakai K, Passingham RE (2006) Prefrontal set activity predicts rule-specific neural processing during subsequent cognitive performance. J Neurosci 26:1211-1218.

Schmidt T, Vorberg D (2006) Criteria for unconscious cognition: three types of dissociation. Percept Psychophys 68:489-504.

Vorberg D, Mattler U, Heinecke A, Schmidt T, Schwarzbach J (2003) Different time courses for visual perception and action priming. Proc Natl Acad Sci USA 100:6275-6280. 